\title{
Inserción laparoscópica de catéter de diálisis peritoneal: experiencia de 3 años*
}

\author{
Drs. FRANCISCO DUBOURNAIS R. ${ }^{1}$, Ints. CAMILA ROJAS S. ${ }^{2}$, NICOLÁS MUÑOZ P. ${ }^{2}$, \\ NICOLÁS GARCÍA S. ${ }^{2}$, ENF. IVANIA BUSTAMANTE V. ${ }^{3}$
}

\author{
Servicio de Cirugía, Hospital Regional de Talca. \\ Interno de Medicina, Universidad Católica del Maule. \\ Enfermera Jefe del Programa de Diálisis Peritoneal, Hospital Regional de Talca.
}

Talca, Chile.

\begin{abstract}
\section{Laparoscopic placement of peritoneal dialysis catheters. Experience in 81 patients}

Background: Laparoscopic placement of peritoneal dialysis catheters is associated with a lower rate of complications. Aim: To report the experience in laparoscopic placement of peritoneal dialysis catheters. Material and Methods: Review of medical records of 81 patients aged 15 to 90 years (42 males) subjected to the procedure between 2010 and 2013. Results: A three port plus omentopexy technique was used. Surgical time was 51 minutes. Other simultaneous surgical procedures were performed in four patients. No patient died, had a bowel perforation or required conversion to open surgery. Ninety eight percent of patients were discharged less than 24 hours after the procedure, using standard analgesics. Four patients required a new surgical procedure due to minor complications. All installed catheters were functional until the moment of his review. Conclusions: Laparoscopic placement of peritoneal dialysis catheters is a safe and effective procedure.
\end{abstract}

Key words: Peritoneal dialysis, catheter, laparoscopic surgery.

\section{Resumen}

Objetivo: Describir la técnica quirúrgica usada en Hospital Regional de Talca (HRT) para la inserción laparoscópica de catéter de diálisis peritoneal y analizar sus resultados. Material y Método: Se realizó un estudio retrospectivo, descriptivo, mediante la revisión de fichas clínicas del total de pacientes sometidos a inserción laparoscópica de catéter de peritoneodiálisis en el HRT entre marzo de 2010 y abril de 2013. Se recopiló información sobre los pacientes, la técnica quirúrgica y complicaciones postoperatorias. Resultados: Total 81 pacientes, sin exclusiones, sometidos a cirugía bajo técnica laparoscópica de 3 puertos más omentopexia. Tiempo quirúrgico medio 50,9 min. Se realizó otro procedimiento quirúrgico simultáneo en 4 pacientes. No se reportaron perforación de víscera hueca ni conversión a cirugía abierta. No hubo mortalidad asociada al acto quirúrgico. El 97,5\% tuvo alta quirúrgica antes de las 24 h postoperatorias, con requerimientos analgésicos

*Recibido el 13 de mayo de 2013 y aceptado para publicación el 3 de junio de 2013.

Los autores no refieren conflictos de interés.

Correspondencia: Dr. Francisco Dubournais R.

Av. San Miguel 3605. Casilla 617. Talca, Chile. Fax: 5671203435

fdubournais@ucm.cl 
estándar. Sólo 4 pacientes requirieron reintervención quirúrgica por complicaciones menores y en 4 se realizó laparoscopia diagnóstica tardía por dolor. El 100\% de los catéteres instalados bajo esta técnica permanecen funcionales hasta el momento del corte de la serie. Conclusión: Nuestra experiencia es comparable con otras series a nivel mundial, siendo un procedimiento reproducible de manera segura y costo-efectiva.

Palabras clave: Diálisis peritoneal, laparoscopia, colocación laparoscópica de catéter.

\section{Introducción}

La Insuficiencia Renal Crónica (IRC) es una patología frecuente tanto a nivel mundial como en nuestro país, con una prevalencia del $2,7 \%$ de la población de 15 años o más ${ }^{1}$. Hasta 2010 el método de sustitución renal más usado en Chile fue la hemodiálisis, sin embargo, luego de la inclusión de la diálisis peritoneal como opción en las Garantías Explícitas en Salud (GES), ha existido un progresivo aumento del número de los pacientes que optan por esta modalidad ${ }^{2}$.

La diálisis peritoneal ha sido probada como un método seguro y efectivo como terapia de reemplazo renal, otorgando mayor autonomía y mejor calidad de vida para el paciente y su familia y existiendo además evidencia suficiente que demuestra que tanto la morbilidad como la mortalidad asociada al acceso es significativamente menor que la hemodiálisis.

En la actualidad, son varios los métodos disponibles para la instalación de catéter de peritoneodiálisis: percutánea, laparotomía, peritoneoscopia e inserción por vía laparoscópica ${ }^{4,5}$.

Tradicionalmente, la técnica más usada consiste en la inserción a ciegas del catéter a través de una pequeña laparotomía abdominal baja ${ }^{6}$, con o sin control fluoroscópico. Existen múltiples estudios prospectivos que muestran ventajas de la técnica laparoscópica en relación a menor tasa de infecciones, fugas, disfunción del catéter, mayor acceso funcional inmediato y una menor tasa de revisión y reemplazo del catéter, además de mayor costo-efectividad en comparación con la técnica tradicional ${ }^{6-9}$. Sin embargo, hasta donde los autores conocemos, no existiría evidencia suficiente para recomendar una técnica por sobre otra.

En Chile, hasta ahora, no existen reportes de la inserción de catéter de diálisis peritoneal con la técnica descrita en este trabajo.

El objetivo de nuestro estudio es describir la técnica quirúrgica del Hospital Regional de Talca para la inserción laparoscópica de catéter de peritoneodiálisis y sus resultados.

\section{Material y Método}

Se realizó un estudio descriptivo, retrospectivo, que incluyó al total de pacientes sometidos a cirugía de instalación de catéter de peritoneodiálisis por vía laparoscópica, en el período comprendido entre marzo de 2010 y abril de 2013, en el Hospital Regional de Talca.

Se recopiló la información relevante para el estudio a través de la revisión sistemática de las fichas clínicas y de registros internos del programa de peritoneodiálisis de nuestro Hospital. Los datos incluyeron aspectos del paciente como edad al momento de la cirugía, sexo, IMC, antecedentes de cirugías abdominales, causa de la IRC, antecedente de hemodiálisis previa y criterio de inclusión al programa de peritoneodiálisis; aspectos de la cirugía como técnica quirúrgica, duración, complicaciones intraoperatorias y estadía hospitalaria; complicaciones postoperatorias precoces y tardías, y sobrevida del catéter hasta abril de 2013.

\section{Técnica}

Bajo anestesia general, en posición decúbito dorsal y previa marcación de los cuffs en la piel del paciente, se realizó neumoperitoneo con aguja de Veres en hipocondrio derecho, insuflando $\mathrm{CO}_{2}$ a un litro por minuto hasta $13 \mathrm{mmHg}$. Exploración de la cavidad abdominal con óptica de $5 \mathrm{~mm}, 30^{\circ}$. De haber adherencias que bloqueen la posición adecuada del catéter, se liberan con Ligasure ${ }^{\circledR}$ o electrobisturí, a través de trocar de $5 \mathrm{~mm}$ en fosa ilíaca derecha. De estar libre la cavidad, se introduce un segundo trocar de $8 \mathrm{~mm}$, paramediano izquierdo extraperitoneal, ingresando a cavidad peritoneal en la pelvis (Figura 1) previo ojal hecho con electrobisturí a través de dicho trocar de $5 \mathrm{~mm}$, instalado en fosa ilíaca derecha. Se introduce catéter Swan Neck Coil $®$ izquierdo, dejando coil en fondo de saco de Douglas. Tunelización subcutánea del catéter, exteriorizándolo en fosa ilíaca izquierda, dejando cuff extraperitoneal y subcutáneo. Prueba de flujo y reflujo. Posteriormente se realiza omentopexia a hipocondrio izquierdo con vicryl 0 con aguja EndoClose ${ }^{\circledR}$. Cierre de incisión paramediana con vicryl 0 , y el resto de las incisiones con nylon. Se usó profilaxis antibiótica, Cefazolina 1 gr preoperatorio, y analgesia postoperatoria estándar con Paracetamol $500 \mathrm{mg}$ cada 8 h vía oral y Paracetamol/Tramadol (375 mg/37,5 mg) si dolor en EVA $>3$.

\section{Estadística}

Para el procesamiento de datos se utilizó el programa estadístico PASW Statistics 18. 


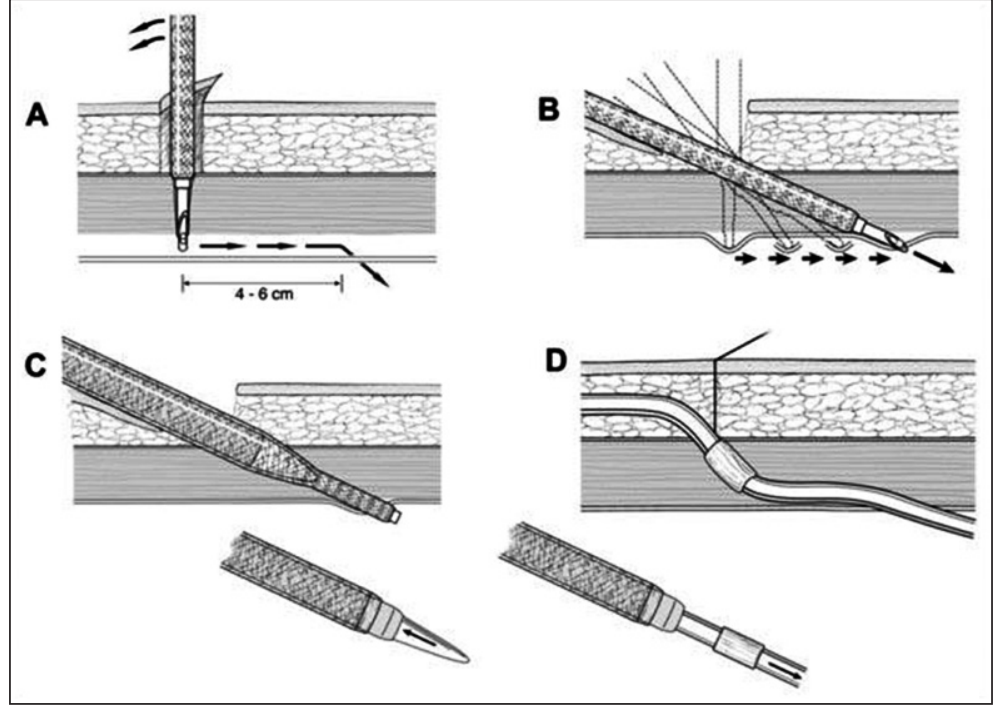

Figura 1. Tunelización extraperitoneal del trocar paramediano izquierdo, previa inserción del catéter, que ingresa a peritoneo en la pelvis bajo visión laparoscópica.

\section{Resultados}

El estudio incluyó un total de 81 pacientes, en los que se realizó un total de 85 procedimientos. El promedio de edad fue 47,1 años (rango de 15-90 años). El $51,9 \%$ fueron hombres $(n=42)$. La principal causa de IRC fue la nefropatía hipertensiva (42\%). Un $56,7 \%$ se encontraba en hemodiálisis previo al ingreso al programa de diálisis peritoneal, 78,3\% lo hacía a través de un acceso vascular definitivo. Del total de pacientes que ingresó al programa, el $84 \%$ lo hizo por opción personal, 9,9\% por agotamiento de capital venoso y un 3,7\% por ruralidad extrema. El resumen de las características generales de la población estudiada se presenta en la Tabla 1.

El IMC promedio de la población fue 25,3 $\pm 4,5$ $\mathrm{kg} / \mathrm{m}^{2}$. El 44,4\% tenía antecedente de cirugía abdominal, siendo la cesárea la más frecuente $(37,7 \%)$.

El tiempo quirúrgico medio fue de 50,9 $\pm 19,1$ minutos. No fue posible la omentopexia en el $14,8 \%$ de los pacientes. Se realizó reparación de hernias a 3 pacientes y colecistectomía, por colelitiasis sintomática, a 1 paciente. No se reportaron perforación de víscera de hueca ni conversión a cirugía abierta. No hubo mortalidad asociada al acto quirúrgico.

Respecto al postoperatorio, el $100 \%$ de los pacientes se realimentó precozmente (antes de $6 \mathrm{~h}$ ). El $97,5 \%$ tuvo alta quirúrgica antes de las $24 \mathrm{~h}$ postoperatorias. El 19,8\% debió permanecer hospitalizado por patologías médicas de base, ruralidad extrema o complicaciones quirúrgicas inmediatas $(\mathrm{n}=1)$.

Del total de pacientes, requirieron reexploración 8 de ellos. Cuatro presentaron complicaciones inmediatas $(<6$ semanas postoperatorias): 2 por obstrucción del catéter, el primero de la serie por omento, luego de lo cual se mejora la técnica con la realización de omentopexia; el segundo por apéndice omental, que requirió liberación, resección con Ligasure y reposicionamiento del catéter. 2 pacientes presentaron hemoperitoneo, sin compromiso hemodinámico, de cuantía leve a moderada, siendo rein-

Tabla 1. Características generales de los pacientes sometidos a inserción laparoscópica de catéter de peritoneodiálisis en el Hospital Regional de Talca, período 2010-2013

\begin{tabular}{|lc|}
\hline Característica & Resultado \\
Total de pacientes & 81 \\
Edad (años) & $47,1 \pm 17,9$ \\
Sexo (\% hombres) & 51,9 \\
IMC $\left(\mathrm{kg} / \mathrm{m}^{2}\right)$ & $25,2 \pm 4,5$ \\
Antecedente cirugía abdominal (\%) & 44,4 \\
Causa IRC (\%) & \\
Hipertensión arterial & 42,0 \\
Glomerulopatía & 13,6 \\
$\quad$ Diabetes mellitus & 12,3 \\
No precisada & 8,6 \\
$\quad$ Otros & 23,5 \\
Indicación diálisis peritoneal (\%) & \\
$\quad$ Electiva & 84,0 \\
$\quad$ Agotamiento capital venoso & 9,9 \\
$\quad$ Ruralidad extrema & 3,7 \\
Otra & 2,5 \\
\hline
\end{tabular}

IMC: Índice de Masa Corporal, IRC: Insuficiencia Renal Crónica. 
tervenidos al primer y cuarto día postoperatorio respectivamente. Estas complicaciones se presentaron al inicio de la serie, cuando el trocar de $8 \mathrm{~mm}$ era introducido al peritoneo por punción. Posterior a ello se modificó la técnica a ojal con electrobisturí, sin nuevos reportes. Durante el seguimiento se detectó dolor al final del drenaje que no cedió a analgesia habitual en 4 pacientes, 3 de las cuales eran mujeres, asociado a aumento de tamaño uterino, y un paciente trasplantado renal. Todos ellos se sometieron a laparoscopia exploradora y reposicionamiento del catéter a fosa ilíaca izquierda fijado con un punto de nylon monofilamento (Ethilon ${ }^{\circledR}$ ), con evolución satisfactoria.

El 100\% de los catéteres instalados por vía laparoscópica fueron funcionales hasta el momento del corte del estudio.

\section{Discusión}

La técnica laparoscópica empleada en nuestra experiencia se realiza en un tiempo similar e incluso menor al descrito en otras series ${ }^{10}$, permite el tratamiento de patologías quirúrgicas coexistentes y la no exclusión de pacientes con antecedentes de cirugías abdominales, obesidad ni poliquistosis renal. A pesar que se presentaron complicaciones, éstas fueron poco frecuentes y sin repercusión sistémica. Otras, descritas en la literatura, como perforación de víscera hueca, conversión a cirugía abierta, migración del catéter, infección de herida operatoria y filtración alrededor del orificio de salida ${ }^{11-13}$ no se reportaron, así como tampoco hubo mortalidad asociada al procedimiento. Los pacientes presentaron un postoperatorio satisfactorio, con una hospitalización abreviada y con requerimientos analgésicos estándar.

Aún con las limitaciones propias de una investigación realizada en sólo un centro asistencial y por el mismo cirujano, concluimos que la inserción laparoscópica del catéter de peritoneodiálisis bajo la técnica quirúrgica empleada descrita, es comparable con otras series publicadas, además de ser un procedimiento reproducible de manera segura y costo-efectiva.

\section{Agradecimientos}

Agradecemos al equipo del Programa de Diálisis Peritoneal del Hospital Regional de Talca por facilitar la realización de esta investigación.

\section{Referencias}

1. Encuesta Nacional de Salud 2009-2010. Disponible en http://epi.minsal.cl/wp-content/uploads/2012/07/ Informe-ENS-2009-2010.-CAP-5_FINALv1julioccepi. pdf (Consultado el 03 de Mayo de 2013).

2. Ministerio de Salud. Guía Clínica Diálisis Peritoneal. Santiago: Minsal, 2010.

3. Mujais S, Holmes C. Modern peritoneal dialysis: concepts and approaches. Kidney Int Suppl, 2006;103:S1-2.

4. Giannattasio M, De Maio P, La Rosa R, Balestrazzi A. Videolaparoscopy: A new alternative for implantation of peritoneal catheters in ESRD patients with previous abdominal surgeries. Perit Dial Int. 1996;16:96-7.

5. Brandt C, Franceschi D. Laparoscopic placement of peritoneal dialysis catheter in patients who have undergone prior abdominal operations. J Am Coll Surg. 1994;178:515-6.

6. European Guidelines on Best Practice for the Management of Peritoneal Dialysis. European Renal Association. Oxford University Press: Baxter Healthcare, 2002.

7. Tsimoyannis E, Siakas P, Glantzounis G, Toli C, Sferopoulos G, Pappas M, et al. Laparoscopic placement of the Tenckhoff catheters for peritoneal dialysis. Surg Laparosc Endosc Percutan Tech. 2000;10:218-21.

8. Gajjar A, Rhoden D, Kathuria P, Kaul R, Udupa A, Jennings WC. Peritoneal dialysis catheters: laparoscopic versus traditional placement techniques and outcomes. Am J Surg. 2007;194:872-6.

9. Crabtree J, Kaiser K, Huen I, Fishman A. Cost-effectiveness of peritoneal dialysis catheter implantation by laparoscopy versus by open dissection. Adv Perit Dial. 2001;17:88-92.

10. Jwo SC, Chen KS, Lee CC, Chen HY. Prospective Randomized Study for Comparison of Open Surgery with Laparoscopic-Assisted Placement of Tenckhoff Peritoneal Dialysis Catheter - A Single Center Experience and Literature Review. J Surg Res. 2010;159:489-96.

11. Hodgson D, Rowbotham C, Peters JL, Nathan S. A novel method for laparoscopic placement of Tenckhoff peritoneal dialysis catheters. BJU International. 2003;91:885-6.

12. Fleisher AG, Kimmelstiel FM, Lattes CG, Miller RE. Surgical complications of peritoneal dialysis catheters. Am J Surg. 1985;149:726-9.

13. Hagen S, van Alphen A, Ijzermans J, Dor F. Laparoscopic versus open peritoneal dialysis catheter insertion, the LOCI-trial: a study protocol. BMC Surg. 2011, 20;11:35. doi: 10.1186/1471-2482-11-35. 